\title{
AN INVESTIGATION OF THE FACTOR STRUCTURE OF THE HARVARD GROUP SCALE OF HYPNOTIC SUSCEPTIBILITY, FORM A (HGSHS:A)
}

\section{Christoph Piesbergen and Burkhard Peter}

\author{
University of Munich, Germany
}

\begin{abstract}
In order to investigate the effects of the hypnotic state a standardized hypnosis session was conducted with 144 subjects in a controlled laboratory study. The induction of a hypnotic trance in the German version of the Harvard Group Scale of Hypnotic Susceptibility (HGSHS:A by Shor and Orne, 1962) was tape-recorded and used as the treatment.

The HGSHS:A seems to be a reliable measure of suggestibility and hypnotizability. This is underlined by the consistent results of a factor analysis on the depths of hypnosis that is in agreement with former studies. Descriptive data analyses with a sufficient number of subjects of high and low suggestibility suggest that our hypnosis induction by tape is an effective method of producing a hypnotic trance. Analyses of within-subjects variables did not reveal any valid predictors of hypnotizability, thereby confirming the need of screening instruments such as the HGSHS. Copyright (c) 2006 British Society of Experimental \& Clinical Hypnosis. Published by John Wiley \& Sons, Ltd.
\end{abstract}

Key words: factor structure, hypnosis, suggestibility scales, HGSHS

\section{Measuring suggestibility}

Differences in the ability to get hypnotized or to be susceptible for hypnotic suggestion have been stated early in the history of hypnosis (e.g. Faria, 1819). The history of hypnotizability and suggestibility scales that have been constructed in order to measure the individual extent of this disposition is thus correspondingly long (for an overview cf. Hilgard, 1965, 1967; Krause, 2001).

Besides the principal debate about what hypnosis is and what hypnotic abilities represent, the question of what hypnotizability scales actually measure was again and again the subject of critical discussions (cf. Weitzenhoffer, 1980; Hilgard, 1981; Perry, Nadon and Button, 1992; Kirsch, 1997). Both questions cause each other due to the interdependence between theory and measuring instruments (Woody, 1997). Today, there is agreement on the fact that hypnotic abilities that can be measured with these scales do not represent a one-dimensional unity but are composed of at least two components (Tellegen, 1978-79). This knowledge was gained among others with the help of factor analysis. The corresponding experiments with suggestibility scales have been carried out already quite some time ago (Eysenck and Furneaux, 1945; Das, 1958; Stukat, 1958; Ås and Lauer, 1962; Hammer, Evans and Bartlett, 1963; Moore, 1964; Eysenck, 1989). 


\section{Suggestibility and hypnotizability}

Usually, suggestibility is equated with hypnotizability. According to Bernheim (1888), hypnosis only amplifies a suggestibility that was already more or less present before hypnosis (Weitzenhoffer, 1993). However, since the late Bernheim (e.g. 1917, p.47) made the provoking thesis that there is no hypnosis - 'Il n'y a pas d'hypnotisme' - because suggestibility is all that matters, this equality has been reconsidered again and again. Hypnotizability was finally defined as suggestibility relative to the induction of hypnosis (e.g. Weitzenhoffer, 1980). However, this is merely 'hypnotic suggestibility', not necessary hypnotizability. Strictly speaking, one would always have to determine suggestibility at first without hypnosis and then with hypnosis in order to calculate hypnotizability from the resulting scores; this has not been done yet, maybe also because generally, suggestibility without hypnosis correlates highly with suggestibility after induction of hypnosis, with correlation coefficients between 0.66 and 0.99 .

Recently, Braffman and Kirsch (1999) calculated the pre-hypnotic suggestibility out of the hypnotic suggestibility and then tried to determine the 'resulting' hypnotizability. Twenty-nine per cent of the subjects did not show any change. Among them were also so-called highly suggestible people, meaning that they do not necessarily have to be highly hypnotizable: if a person that is already very suggestible without hypnosis does not experience any further increase of his or her hypnotic abilities, one can conclude that the person is highly suggestible but nevertheless not at all hypnotizable. With $46 \%$ of the subjects, the usual improvement of the reactions to suggestions appeared and $25 \%$ of the subjects showed a reduction of (pre-hypnotic) suggestibility. This means that the induction of hypnosis can sometimes also be detrimental.

Until now, it did not gain acceptance consequently to make this division into suggestibility and hypnotizability. We therefore abstain from it, especially since our experiment was carried out before 1999.

\section{The three components theory of suggestibility and hypnotizability}

It has been questioned again and again whether suggestibility and hypnotizability would be one single dimension like the different scales pretend to measure (for an overview, cf. Krause, 2001, p.116). After a variety of examinations that analysed factors, distributions, profiles and clusters (e.g. Balthazard and Woody, 1992; Balthazard, 1993; Pekala and Forbes, 1997) there is still no consent on the number of components that are measured by the different scales.

Peter (2001, p.44ff) has organized the different hypnotic phenomena into motoric/ kinaesthetic, sensory/affective, and cognitive, according to the phenomenological aspects. From clinical experience, it is obvious that the motoric items are significantly easier to be executed, i.e. they can be successfully executed by considerably more people - and they are also more likely to be modifiable through the different non-state factors such as, for example, compliance or training - than the sensory and cognitive items which are seen as the so-called 'pure' hypnosis tasks by some authors (Woody, Bowers and Oakman, 1992). It seems that even the most notorious among the social psychological hypnosis researchers, Nicholas Spanos, shares this opinion, since he writes that 'cooperation and expectation can be significant especially during the reaction to ideomotoric and challenge suggestions while the ability to consider imaginations for real [. . .] becomes increasingly important for the "cognitive" items' (Spanos et al., 1980, quoted from 
Balthazard, 1993, p.56; cf. also Spanos, 1989). In this context, one should also remember that during the times of James Braid - in the middle of the $19^{\text {th }}$ century - hypnosis was only considered to be 'real' when a spontaneous posthypnotic amnesia occurred afterwards (Braid, 1843). One could conclude from these experiences and considerations that suggestibility and hypnotizability are at least two components, one motoric/kinaesthetic and the other one sensory/affective.

If we take a closer look at the motoric tasks of the Harvard and the Stanford Scales, we can see that they are also different. There are tasks that suggest a letting go or giving up of despotism ('you cannot move the hand/the arm, open the eyes, not talk anymore', etc.) and tasks which suggest unvoluntary movements ('Your head drops to the front, your eyes close, your hand [that was lifted arbitrarily before] comes down on the armrest again', etc.).

Appropriately, a factor analysis that was already carried out in 1965 by Hilgard on the SHSS:A with 402 subjects resulted in three unrotated factors which explained $53 \%, 10 \%$ and $6 \%$, thus altogether $69 \%$ of the variance; further factors only explained less than $3 \%$. The first factor concerns those motoric/kinaesthetic tasks (finger lock, eye catalepsy, arm immobilization, inhibition, arm rigidity) that challenge character and demand a 'loss of arbitrary control'; the five items of this factor load between 0,85 and 0,90 on this first factor. On the second factor, the motoric tasks that charge most highly are those that are appealed to with direct suggestions (hand lowering, hands moving, eye closure, and the body swing test) and where the type of the tasks demands the principal permission of unvoluntariness. The third factor finally relates to sensory and cognitive tasks (hallucination, posthypnotic suggestion and amnesia).

Peters and colleagues (1974) have conducted a factor analysis in two examinations about the HGSHS:A and came to similar results as Hilgard (1965): factor 1 refers to arm immobilization, finger lock, arm rigidity, communication inhibition, and eye catalepsy; factor 2 refers to head falling, hand lowering, and hands moving; factor 3 concerns hallucination and posthypnotic suggestion (the amnesia item was left out).

These two and further, earlier examinations (Hammer et al., 1963; Evans, 1965) show that suggestibility/hypnotizability is by no means only one dimension or characteristic, but that at least three factors have to be differentiated. It is not possible to decide here to what an extent one should differentiate again between the sensory and the cognitive tasks since the Harvard and the Stanford Scales include only few of these tasks, at least only a few compared to the number of motoric/kinaesthetic tasks.

\section{Questions and hypothesis of the present examination}

Due to these examples, the authors have conducted a controlled laboratory study that was supposed to create constant and therefore comparable conditions for a sample $(\mathrm{N}=$ 144) during a standardized hypnosis session. The tape-recorded spoken text of the German version of the 'Harvard Group Scale of Hypnotic Susceptibility' (HGSHS by Shor and Orne, 1962; German version by Walter Bongartz, 1982) was used as treatment. Further, comparisons between anxious and less anxious test subjects (measured with STAI-X-2; Laux et al., 1981) and between people that have an ability for absorption and people that are stress susceptible (measured by the Differential Personality Questionnaire, DPQ by Tellegen, 1978-79; Tellegen and Atkinson, 1974; German version by Hansjürgen Meyer, 1982) were made. The following conclusions were drawn from these and further questions: 
- There are differences of hypnotizability between men and women.

- There are differences of hypnotizability due to handedness (connected to the hemisphere dominance).

- There are differences of hypnotizability between 'day and night persons' (due to different sleeping - waking rhythms).

- There are differences of hypnotizability with people who had miscellaneous previous experiences with relaxation methods and hypnosis.

- The distribution of the feature 'hypnotizability'/'suggestibility' corresponds to a normal distribution whereby $10 \%$ of extremely highly suggestible and extremely lowly suggestible people have to be isolatable.

- Clustering of the HGSHS(A) items regarding contents is possible and corresponds to the order of the American original and the phenomenological presentation by Peter (2001).

\section{Sample and course of the experiment}

The test persons were either recruited with posters and flyers by the Milton Erickson society for clinical hypnosis (MEG) in Munich, or they came from the circle of friends of the experimenters or were recruited from passers-by that were approached ad hoc. The youngest test person was 20 , the oldest 67 years old. The sex ratio was $38 \%$ men/ $62 \%$ women, 132 test persons were right-handed, 9 were left-handed and ambidextrous or not classifiable were 3 persons. The characteristic of sleeping/waking cycle presented a better ratio in the sense of a uniform distribution: 30 persons called themselves early risers opposed to 57 night persons and another 57 did not want to give definite information about this characteristic. Earlier experiences with hypnosis, meditation or other relaxation methods were also determined in order to be able to control eventual influences on hypnotizability. Eighteen test persons had experiences with hypnosis, 51 with meditation and 86 with relaxation methods.

The experiment took place in the psycho-physiological laboratory of the department of psychology of the University of Munich. These laboratories are nearly hermetically protected from noise, smell, temperature fluctuations and partly even from electromagnetic radiation so that no interfering variables concerning these aspects were expected. The equipment consisted of a relaxation chair for the test person, a customary PC for the input of the answers to the psychometric tests and a stereo with a tape deck for playing the hypnosis instruction. The experiment room was separated from the foyer by a well-closing door where the experimenters stayed during the experiment. They were connected to the experiment room through an overhearing device in order to be able to intervene immediately in case of eventual incidents. First, the test person filled in the $\mathrm{X}-2$ and the DPQ questionnaire on the PC. The experimenter then placed the subject on the chair and started the tape with the hypnosis induction from the HGSHS:A. The test person was then left alone for approximately 50 minutes and treated at the end of the session to the questionnaire on the HGSHS:A.

\section{Measuring instruments}

Although the experimental setting of this study was planned as single testing, we decided to use the The Harvard Group Scale of Hypnotic Susceptibility HGSHS (form A) since its execution is more economical (shorter) and numerous items from the SHSS are not practicable for the additionally planned derivation of special physiological parameters 
due to the fixation of the left hand. Since the HGSHS has been developed from the SHSS, both scales are very similar (Shor and Orne, 1963). Nevertheless, we had to modify the HGSHS:A as well: item numbers 5 and 7 of the original version, consisting of a finger lock and hands moving were removed because they were not feasible due to the derivation of physiological parameters; the hallucination of a flying mosquito was changed into the hallucination of a fly for our degrees of latitude and the eye catalepsy preceded this item. The text to the individual tasks was then read out by an experienced hypnotherapist and recorded on tape. Table 1 gives an overview on the order of the items and the temporal structure of the recorded text.

Further, the 'State-Trait Anxiety Inventory' (STAI) by Spielberger that has been translated to German and validated by Laux, Glanzmann, Schaffner and Spielberger (1981) was used, as well as the Tellegen Differential Personality Questionnaire (DPQ) (Tellegen, 1978-79; German translation by Meyer, 1982). Tellegen and Atkinson (1974) showed that absorption as the ability to be completely involved in a real and imaginative activity has a very close relation to suggestibility. In their work, they compare the already mentioned Stanford Hypnotic Susceptibility Scale (SHSS; Weitzenhoffer and Hilgard, 1959), which is a direct measure of a person's reaction to hypnotic suggestion, with the test for

Table 1. Timer of the hypnosis examination

\begin{tabular}{|c|c|c|}
\hline Minute & Instruction and description of the task & Item $(\mathrm{Nr})$ \\
\hline $1-2$ & Introducing words & Introduction \\
\hline 3 & Head lifting, keeping it lifted / closing the eyes; & (1) Head falling \\
\hline $4-5$ & Head falls / head falls further down; & \\
\hline $6-7$ & Sitting back / opening the eyes / fixation / relaxation. & \\
\hline $8-11$ & Stressing of cooperation, concentration etc. / fixation; & (2) Eye closure \\
\hline $12-18$ & Relaxation / heaviness of the eyelids / the eyes close; & \\
\hline $19-23$ & Suggestion of sleep / induction by counting / break. & \\
\hline $24-25$ & Bringing the attention to the hand (with extended arm); & (3) Hand lowering \\
\hline 26 & Suggestion of heaviness and the sinking down of the hand; & \\
\hline $27-29$ & $\begin{array}{l}\text { Sinking down of the hand (with counting) / hand, arm back / } \\
\text { relaxation. }\end{array}$ & \\
\hline 30 & Suggestion of heaviness, immovability of the arm; & (4) Arm \\
\hline $31-32$ & Request to check / relaxation / stretching the arm. & immobilization \\
\hline 33 & Clenching the fist / suggestion of stiffness of the arm; & (5) Arm rigidity \\
\hline $34-35$ & Suggestion of stiffness ('iron bar') / short break / relaxation. & \\
\hline 36 & Relaxation / suggestion of inability to shake the head; & (6) Communication \\
\hline 37 & Break / relaxation / request to shake the head. & inhibition \\
\hline 38 & Eyes closed / suggestion of inability to open the eyes; & (7) Eye catalepsy \\
\hline $39-40$ & Inability to open the eyes / request for it / break. & \\
\hline 41 & Hallucination of a fly / request to scare the fly away; & (8) Hallucination \\
\hline 42 & Fly goes away / relaxation / explanation. & \\
\hline 43 & Continuing the explanation / suggestion of forgetting; & (9) Posthypnotic \\
\hline 44 & Setting up the moment of remembering; & suggestions \\
\hline $45-46$ & Suggestion of touching the ankles / counting / knocking. & \\
\hline 47 & Break / explanation of the questionnaire / from now on & (10) Amnesia \\
\hline $48-50$ & $\begin{array}{l}\text { artifacts of movements; filling out the questionnaire / } \\
\text { abolition of amnesia. }\end{array}$ & \\
\hline 51 & Filling out the rest of the questionnaire. & End \\
\hline
\end{tabular}


measuring the more universally valid characteristics of personality that they had developed. Among the main characteristics, stability, introversion and absorption, they only found a correlation between absorption and hypnotizability. Other authors also discuss a relation between absorption and the hypnotic state (Jean and MacLeod, 1983; Pekala, Wenger and Levine, 1985) or its correlation with children (Plotnick, Paine and O'Grady, 1991).

\section{Results}

\section{Descriptive analyses and correlations}

The X-2-test for measuring timidity had low results with 12 persons, medium results with 70 persons and high results with 47 test persons. The DPQ showed low scales with 17, medium scales with 59 and high scores with 54 subjects concerning the ability for absorption. Fifty-nine subjects turned out to be little susceptible to stress, 54 to be medium and 17 to be strongly susceptible to stress. The hypnotizability indicators were formed from the sum of the items of the HGSHS:A that were solved according to the subjects. Fourteen persons fell into the group 'low' (0-2 items solved), 66 into the group 'medium' (3-5 items solved), 55 into the group 'medium high' (6-8 items solved) and 7 into the group 'very high' (9-10 items solved). Thus, the distribution is as expected, with very few lowly suggestible and even fewer highly suggestible subjects.

A further examination of the descriptive statistical values shows that, on an average, the subjects remembered approximately 4 tasks $(\mathrm{M}=3.85 ; \mathrm{s}=1.77 ; \min =0 ; \max =7$; $\mathrm{n}=142)$, regarded approximately half of the tasks as solved $(\mathrm{M}=5.22 ; \mathrm{s}=2.22 ; \mathrm{min}=$ $0 ; \max =10 ; \mathrm{n}=142)$ and the complete mean average value of the experienced depth of the hypnosis $(\mathrm{M}=4.69 ; \mathrm{s}=1.77 ; \min =1 ; \max =8.8 ; \mathrm{n}=131)$ is nearly in the middle between 1 and 10. The different sample extents are mostly due to missing information of single subjects for the respective variables.

Figure 1 shows the course of the average assessment of depth of the trance by the complete sample for each item. It is striking that there is a quick increase of the average depth until item 3 and then a more or less continuous decrease even under the base level. After the initial phase of relaxation until the third item follows thus a nearly constantly increasing, subjectively felt cognitive activation whose intensity from the eighth item on even exceeds the initial awake state.

A high and positive correlation between stress susceptibility and timidity $(r=0.73$; $p=0.000 ; n=129)$ indicates a close relationship between these two constructs. A weak positive correlation between stress susceptibility and absorption ability was found $(\mathrm{r}=$ $0.17 ; \mathrm{p}=0.048 ; \mathrm{n}=130)$. A high absorption ability correlates with a higher hypnotizability $(\mathrm{r}=0.24 ; \mathrm{p}=0.003 ; \mathrm{n}=130)$ and with a higher depth of the trance averaging all items $(\mathrm{r}=0.1541 ; \mathrm{p}=0.047 ; \mathrm{n}=120)$. The depth of the trance covariates also significantly positively with the number of solved tasks of the HGSHS:A, thus with suggestibility/ hypnotizability $(\mathrm{r}=0.63 ; \mathrm{p}=0.000 ; \mathrm{n}=131)$, which again meets the expectations. The variations of the sample extents are due to missing data for the respective pair of variables.

\section{Variance analyses of organism and hypnotizability variables}

A four-factorial analysis of the covariance $(2 \times 3 \times 3 \times 4-$ design $)$ did not show any significant differences of the mean with any of the main effects $\operatorname{sex}(F=0.165 ; p=0.685)$, handedness $(F=0.643 ; p=0.527)$, sleeping/waking cycle $(F=2.269 ; p=0.108)$ and previous experience $(\mathrm{F}=0.429 ; \mathrm{p}=0.733)$ for the measured variable hypnotizability. 


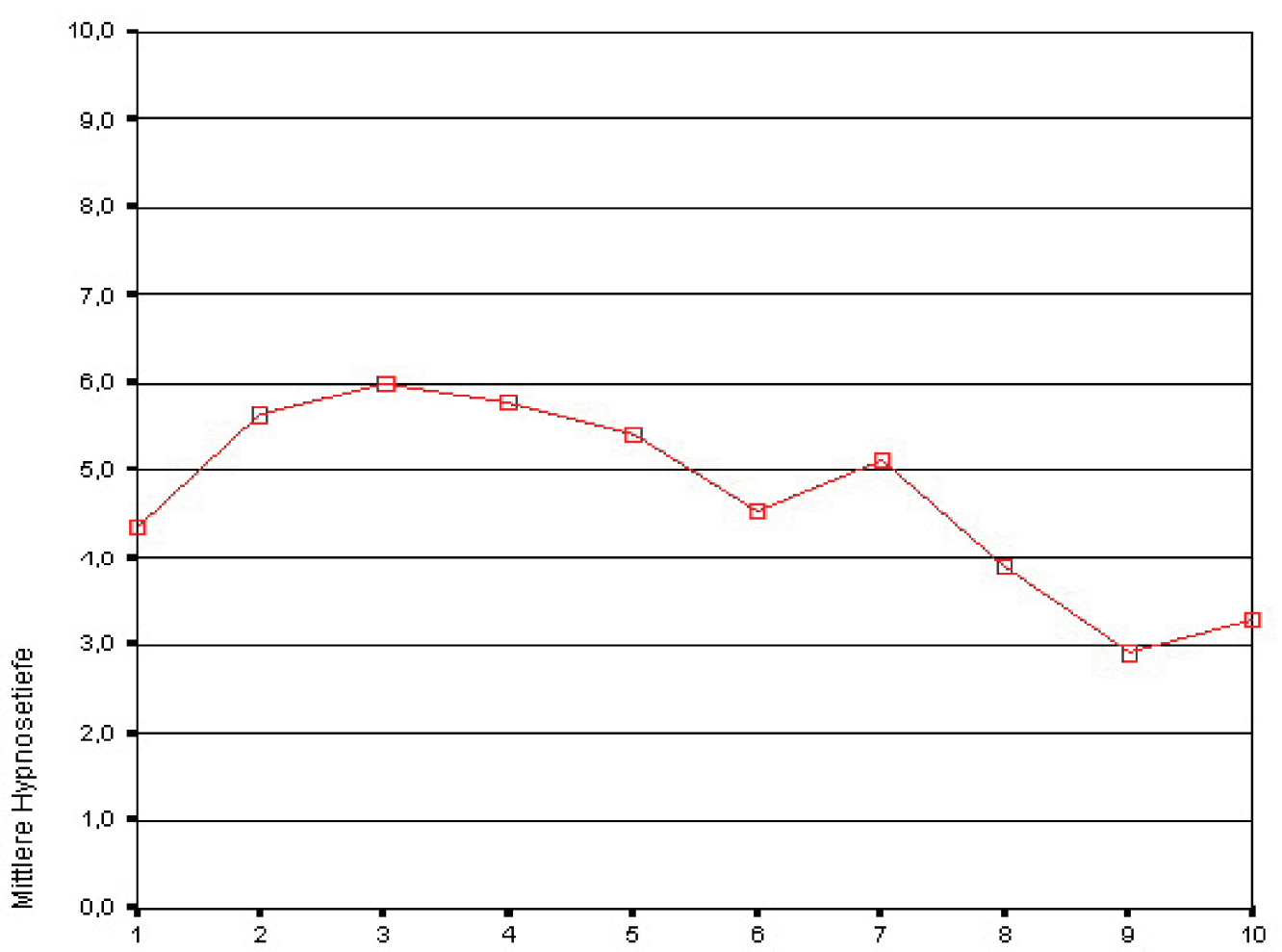

Itemnummer der modifizierten HGSHS.A

Figure 1. Course of the average assessment of the trance depth $(\wedge$ mean average of depth of hypnosis $\rightarrow$ item number of the modified HGSHS:A).

Also, the covariates that were included into the model as assumed interfering variables did not show any significant effects, neither together $(F=1.865 ; p=0.160)$ nor one by one (age: $\mathrm{F}=1.694 ; \mathrm{p}=0.196$; time: $\mathrm{F}=2.402$; $\mathrm{p}=0.124$ ). Before conducting a covariance analysis, the homogeneity of the variance was checked with a Cochran - test $(\mathrm{C}=$ $0.1417 ; p=0.117$ ) and the level of significance was fixed at $5 \%$ for all tests. Referring to the hypothesis 1 with 4 one can therefore assume that sex, handedness, sleeping/waking habits and previous experiences with relaxation methods or hypnosis do not or only coincidentally play a role in differences of hypnotizability.

\section{Factor analysis of the HGSHS:A: items}

Since a linear independence (orthogonality) of the factors was not to be presumed (which would in the end have meant that the factors represent totally different concepts) and in order to see whether our data for the HGSHS:A showed a similar structure to the structure of Peters and colleagues (1974) and of Hilgard (1965) for the SHSS:A, we conducted a principal component analysis followed by an oblique factor rotation ('direct oblimin' with $\delta=0$ ). With the help of the factor analysis, a further validation of the measuring instrument was done because on one side, results from the German-speaking territory are rare and on the other side, it also had to be checked which influences arose from the modifications that we made. 
Since the dichotomous items (solved/not solved) of the HGSHS:A are poorly suitable for a factorization of Pearson's correlation coefficients, we consulted the 10-stages scaled self-assessment of the depth of hypnosis for every item for the factor analysis. In addition, it is also to be expected that a 10-stages scaled assessment provides more information than a dichotomous one. Subjects who showed missing assessments of depth were excluded, so that the calculations were made with $\mathrm{N}=131$. Analogue to the results of Hilgard (1965) for the SHSS:A, three factors could be extracted: the general factor explains $45.5 \%$ of the total variance, factor 2 still $15.2 \%$ and the third factor explains $9.9 \%$. However, factor 3, with 0.99 , does just not fulfil the Eigen value criterion, according to which factors that explain less variance than a single item are actually irrelevant. For reasons of parallelism for the factor analysis of the SHSS:A, it was extracted anyways. In Table 2 , the factor loadings with values above 0.5 are written in italic, those with values above 0.6 are written in bold and thus clarify very well the structure of the HGSHS:A in which the interpretation criterion for a significant factor loading was a value higher than 0.6 .

Factor 1 loads especially high at the depth assessment of those motoric-kinaesthetic items (tasks 4-8), that also form the first factor 'Loss of arbitrary control' in the works of Hilgard (1965) and Peters et al. (1974). Factor 2 is equivalent to factor 2 as found by Hilgard (1965) and by Peters and colleagues (1974); however, we interpret it as 'Introduction to unvoluntariness' (tasks 1-3). Item 4 (arm immobilization) shows a considerable loading on this factor as well and item 3 (hand lowering) loads relatively high on factor 1, too, which makes it seem to be a good transition item from 'Introduction to unvoluntariness' to 'Loss of arbitrary control'. Factor 3 relates to sensory and cognitive tasks (hallucination, posthypnotic suggestions, amnesia), just as with Hilgard. It is to be said that items 8 (hallucination) and 9 (posthypnotic suggestion) show high loadings in factor 1 as well as in factor 3. It is thus through their intermediate positions that they form a good transition from the motoric to the sensory and the cognitive items.

Table 2. Rotated matrix of the factor structure for DEPTH 1 to DEPTH 10

\begin{tabular}{llllll}
\hline Variable & Description & Factor 1 & Factor 2 & Factor 3 & Communality \\
\hline DEPTH 1 & Head falling & 0.19551 & $\mathbf{0 . 8 4 2 7 8}$ & 0.11005 & 0.71269 \\
DEPTH 2 & Eye closure & 0.29009 & $\mathbf{0 . 8 2 1 7 8}$ & 0.37279 & 0.73721 \\
DEPTH 3 & Hand lowering & 0.51935 & $\mathbf{0 . 7 8 6 6 7}$ & 0.10053 & 0.73204 \\
DEPTH 4 & Arm immobilisation & $\mathbf{0 . 7 8 7 5 3}$ & 0.55206 & 0.37592 & 0.74039 \\
DEPTH 5 & Arm rigidity & $\mathbf{0 . 8 1 9 8 5}$ & 0.28094 & 0.21195 & 0.68585 \\
DEPTH 6 & Communication inhibition & $\mathbf{0 . 8 4 2 3 4}$ & 0.21229 & 0.31218 & 0.71051 \\
DEPTH 7 & Eye catalepsy & $\mathbf{0 . 8 3 4 9 2}$ & 0.28996 & 0.48083 & 0.73123 \\
DEPTH 8 & Hallucination & $\mathbf{0 . 6 6 2 6 2}$ & 0.04990 & 0.58579 & 0.59883 \\
DEPTH 9 & Posthypnotic suggestions & 0.50489 & 0.12103 & $\mathbf{0 . 7 9 2 5 7}$ & 0.68165 \\
DEPTH 10 & Amnesia & 0.28485 & 0.29228 & $\mathbf{0 . 8 3 9 0 2}$ & 0.73616 \\
Eigen value and part of the & $\mathbf{F a c t o r} \mathbf{1}$ & $\mathbf{F a c t o r} \mathbf{2}$ & $\mathbf{F a c t o r} \mathbf{3}$ & $\mathbf{t}$ \\
$\begin{array}{l}\text { Overall variance that is explained by the } \\
\text { factors in \% (= Ev } \times 10)\end{array}$ & & & & \\
Before the oblique rotation & $\mathbf{4 . 5 5 0}$ & $\mathbf{1 . 5 2 3}$ & $\mathbf{0 . 9 9 4}$ & $\mathbf{7 . 0 6 7}$ \\
After the oblique rotation & $\mathbf{3 . 8 6 9}$ & $\mathbf{2 . 6 2 1}$ & $\mathbf{2 . 3 4 8}$ & - \\
\hline
\end{tabular}


In the right column of Table 2 , the communalities are listed as the variance of every single item explained by the single factors (maximum $=1.0$ ); they are sufficiently high, with values from 0.6 to 0.75 . The part of the overall variance that is altogether explained before rotation is just over $70 \%$; factor 1 alone explains here also after rotation approximately $39 \%$ of the variance, factor 2 still approximately $26 \%$ and factor 3 approximately $23 \%$. Again, this justifies the retention of a three-factor solution. A summation of the parts of the variance after the skew-angled rotation is, however, not possible.

Figure 2 represents graphically the position of the factors as clusters in the threedimensional space. Cluster 1 therefore consists of the depth assessments for the introduction items (1-3) and corresponds to factor 2 of the main components analysis. Cluster 2 represents the depth of the trance of the hypnosis items (4-8) of the first factor and cluster 3 represents the depth of the trance of the sensory-cognitive tasks of the HGSHS:A (factor 3). The intermediate position of item 4, positioned on the upper margin of cluster 2 and thus also optically documenting its proximity to cluster 1 as regards context, is well recognizable. Similar conditions can be found for item 8 , which, on the right margin of cluster 2, marks the transition to cluster 3. In a purely graphic manner within this cluster, the relative distance between the depth of the hypnosis of items 9 and 10 seems high, which could indicate a marginal or special position of task 10 (amnesia). This is however not interpretable from the diagram.

Therefore, a calculation of the reliability coefficient (Cronbach's $\alpha$ ) was conducted, this time on the dichotomous items (solved/not solved) of the HGSHS:A. Its score of $0.5897(\mathrm{~N}=133)$ seems not to be high. The small difference with the standardized $\alpha=$ 0.5872 indicates a homogenous variance of the items and thus, the reason for the low value seems not to be here. In order to track down the items that we can eventually do without, an analysis of the discriminatory power was conducted.

For this purpose, discriminatory power, the part of the variance $\left(\mathrm{R}^{2}\right)$ explained by the respective item and Cronbach's $\alpha$ if the respective item was eliminated are listed in Table 3. It is striking that especially item 10 is nearly not discriminating at all and that items 1, 3, 8 and 9 fall also below the common limit value of 0.3 . It results that from a

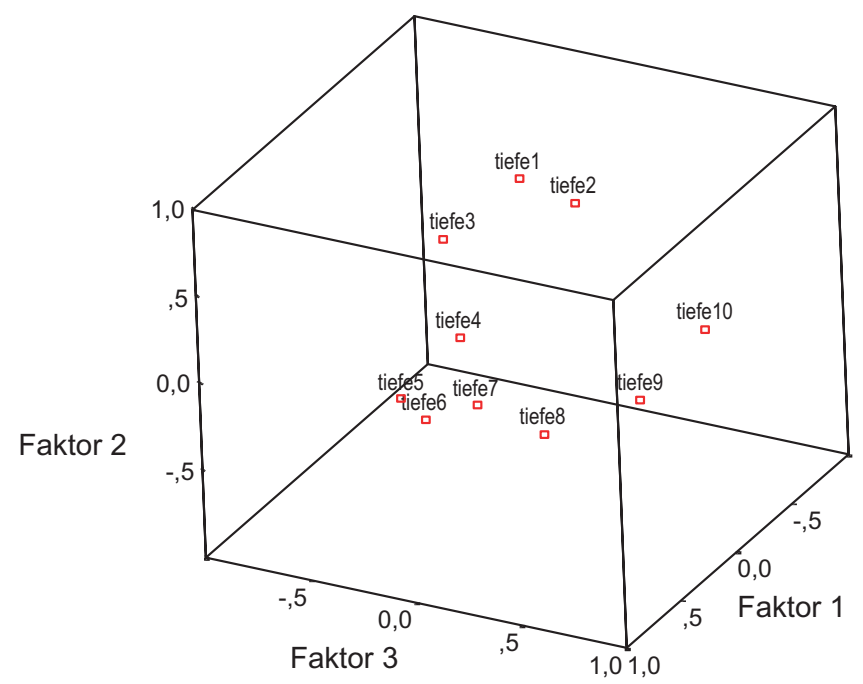

Figure 2. Factors in the rotated space ('tiefe' = depth; 'faktor' = factor). 
Table 3. Discriminatory power coefficients of the HGSHS:A

\begin{tabular}{rlccc}
\hline Item & \multicolumn{1}{c}{ Description } & $\begin{array}{c}\text { Discriminatory } \\
\text { power }\end{array}$ & $\mathrm{R}^{2}$ & $\alpha$ - Item \\
\hline $\mathbf{1}$ & Head falling & 0.1428 & 0.1003 & 0.5931 \\
$\mathbf{2}$ & Eye closure & 0.3548 & 0.1775 & 0.5436 \\
$\mathbf{3}$ & Hand lowering & 0.2018 & 0.2293 & 0.5782 \\
$\mathbf{4}$ & Arm immobilization & 0.3544 & 0.2242 & 0.5403 \\
$\mathbf{5}$ & Arm rigidity & 0.4581 & 0.3167 & 0.5107 \\
$\mathbf{6}$ & Communication inhibition & 0.4106 & 0.3570 & 0.5265 \\
$\mathbf{7}$ & Eye catalepsy & 0.4662 & 0.3256 & 0.5084 \\
$\mathbf{8}$ & Hallucination & 0.2345 & 0.1870 & 0.5714 \\
$\mathbf{9}$ & Posthypnotic suggestions & 0.1393 & 0.1863 & 0.5957 \\
$\mathbf{1 0}$ & Amnesia & -0.0507 & 0.1306 & 0.6407 \\
\hline
\end{tabular}

purely test theoretical point of view, one could do without the amnesia item with a discriminatory power of -0.0507 in any case. With its elimination, the reliability coefficient of the complete test would raise to 0.6407 , which would then even move it into a teststatistically defensible range.

\section{Interpretation of the results}

During the validation examinations of the measure instruments, we found that the use of the DPQ-personality inventory (Tellegen, 1978-79) for the determination of hypnotizability correlates seems not to be very recommendable - at least concerning the stress susceptibility scale. It would have been better to keep the initial TAS-scale which only relates to absorption ability since the construct of stress susceptibility apparently does not form the expected counter-pole to absorption ability and the high correlation with the trait-anxiety make it seem redundant. The test-authors as well (Tellegen and Atkinson, 1974) found only significant coherences between the scale for the absorption ability and the results of the suggestibility scale of Weitzenhoffer and Hilgard (1959).

The high correlation of hypnotizability, as number of solved items in the HGSHS:A, with the medium depth of trance induced us to calculate a factor analysis on the metric scaled estimations of the depth of the hypnosis for the respective items. Thereby, a good reference to the results of Hilgard (1965) and Peters and colleagues (1974) is established. The first factor loads especially high with the depth assessments of the motoric/kinaesthetic hypnosis items that form approximately analogously to Hilgard (1965) or Peters and colleagues (1974) the factor 'Loss of arbitrary control'.

The second factor can correspondingly be seen as the introduction to the unvoluntariness at the beginning of the hypnosis when led from the consciously experienced relaxation to the phenomenon of unvoluntariness. Factor 3 relates to the sensory and cognitive tasks, thus is the same as found by Hilgard. With high double charges at the intersection points, the factor matrix indicates further 'soft' transitions from the introductory phases to the actual hypnosis phase and from there on to the sensory and cognitive phase.

One should, however, not forget that the modification of the HGSHS:A that we undertook limits the broad extrapolatability of the results on the German initial form (Bongartz, 1982) to some extent; this concerns especially the exchange of items 7 and 
8 as opposed to the (also German) original version: in order to have all the motoric items in a row, we placed the hallucination after the eye catalepsy.

An analysis of the intern consistency (Cronbach's $\alpha$ ) on the dichotomous items (solved/not solved) of the HGSHS:A showed that with an elimination of the amnesia item, the reliability coefficient of the complete test would rise from 0.59 to 0.64 which suggests a renunciation of this item. Nevertheless, it seems that the version of the HGSHS:A that we used here has proved itself as measure instrument for suggestibility and hypnotizability. Furthermore, the descriptive data analyses with sufficiently high numbers of highly and lowly suggestible subjects and relative high subjective assessments of the individual hypnosis depth indicate that the method of hypnosis induction from a tape represents a sufficiently strong treatment for the creation of a hypnotic trance.

The variance analyses for the organism variables showed that sex, handedness, sleeping and waking habits and previous experience with hypnosis or relaxation methods barely or only coincidentally play a role concerning differences in hypnotizability. Neither age of the subjects nor time of the examination had a modulating effect on hypnotizability. Apparently, the immanent variables that we set up do not give valid predictors for a pre-estimation of hypnotizability, which confirms the necessity of screening instruments like the HGSHS or the SHSS.

\section{Conclusions}

The following conclusions result from this as well as previously conducted examinations:

- Suggestibility/hypnotizability is not a single, one-dimensional disposition with various expressions (from not hypnotizable via more or less hypnotizable to the hypnotic 'virtuosi') but it consists of at least three different factors.

- Suggestibility/hypnotizability can only be measured with the help of corresponding scales, due to absent or too weak correlations with other dispositions.

- Since suggestibility/hypnotizability represents obviously special and distinctive positions such as intelligence or musicality, it should be taken into account at the use of hypnosis in an experimental or clinical context.

The neo-Ericksonian movement, that is to say pupils of Milton H. Erickson of the second generation suggest fairly often that the trance experience of a given patient is a function of the abilities of the respective hypnotherapist but not of the suggestibility/hypnotizability of this individual patient. This attitude is very unfriendly towards therapists and leads to frustration and eventually to them giving up hypnosis. This could be avoided if more importance was given again to suggestibility/hypnotizability.

It is plausible that in hypnosis experiments, results are only achieved if the subjects are selected according to their suggestibility/hypnotizability and only the highly suggestible are compared to the lowly suggestible.

\section{References}

Ås A, Lauer LW (1962) A factor-analytic study of hypnotizability and related personal experiences. International Journal of Clinical and Experimental Hypnosis 10: 169-82.

Balthazard CG (1993) The hypnosis scales at their centenary: some fundamental issues still unresolved. International Journal of Clinical and Experimental Hypnosis 41: 47-73. 
Balthazard CG, Woody EZ (1992) The spectral analysis of hypnotic performance with respect to 'absorption'. International Journal of Clinical and Experimental Hypnosis 40: 21-43.

Bernheim H (1888) Die Suggestion und ihre Heilwirkung (Übers. von Sigmund Freud). Tübingen: Edition Diskord. (Fotomechanischer Nachdruck der Ausgabe Leipzig und Wien, 1985).

Bernheim H (1917) Automatisme et Suggestion. Paris: Alcan.

Bongartz W (1982) Harvard Group Scale of Hypnotic Susceptibility Form A. Unpublished translation into German, University of Konstanz.

Braffman W, Kirsch I (1999) Imaginative suggestibility and hypnotizability: an empirical analysis. Journal of Personality and Social Psychology 77: 578-87.

Braid J (1843) Neurypnology: Or, the Rational of Nervous Sleep, Considered in Relation with Animal Magnetism. London, Edinburgh: Churchill \& Black.

Das JP (1958) Factor analysis of a hypnotic scale. Indian Journal of Psychology 33: 97-100.

Evans FJ (1965) The structure of hypnosis: a factor analytic investigation. Unpublished doctoral dissertation, University of Sydney.

Eysenck HJ (1989) Personality, primary and secondary suggestibility, and hypnosis. In: VA Gheorghiu, P Netter, HJ Eysenck, R Rosenthal (eds) Suggestion and Suggestibility: Theory and Research. Berlin: Springer, 57-68.

Eysenck HJ, Furneaux WD (1945) Primary and secondary suggestibility: an experimental and statistical study. Journal of Experimental Psychology 35: 485-503.

Faria JC (1819) De la Cause du Sommeil Lucide: Ou, Etude sur la Nature de l'Homme (ed. DG Dalgado, 2nd edition, 1906). Paris: Henri Jouve.

Hammer AG, Evans FJ, Bartlett M (1963) Factors in hypnosis and suggestion. Journal of Abnormal Social Psychology 67: 15-23.

Hilgard ER (1965) Hypnotic Susceptibility. New York: Harcourt.

Hilgard ER (1967) Individual differences in hypnotizability. In: JE Gordon (ed) Handbook of Clinical and Experimental Hypnosis. New York: Macmillan, 391-43.

Hilgard ER (1981) Hypnotic susceptibility scales under attack: an examination of Weitzenhoffer's criticisms. International Journal of Clinical and Experimental Hypnosis 29: 24-41.

Jean RS, MacLeod C (1983) Hypnosis, absorption, and time perception. Journal of Abnormal Psychology 92: 81-6.

Kirsch I (1997) Suggestibility or hypnosis: what do our scales really measure? International Journal of Clinical and Experimental Hypnosis 45: 212-25.

Krause C (2001) Hypnotisierbarkeit, Suggestibilität und Trancetiefe. In: D Revenstorf, B Peter (Hrsg) Hypnose in Psychotherapie und Psychosomatik und Medizin. Ein Manual für die Praxis. Heidelberg: Springer, S.101-19.

Laux L, Glanzmann P, Schaffner, Spielberger CD (1981) Das State - Trait - Angstinventar, Theoretische Grundlagen und Handanweisung. Weinheim: Beltz.

Moore RK (1964) Susceptibility to hypnosis and susceptibility to social influence. Journal of Abnormal Social Psychology 68: 282-94.

Pekala RJ, Forbes EJ (1997) Types of hypnotically (un)susceptible individuals as a function of phenomenological experience: towards a typology of hypnotic types. American Journal of Clinical-Hypnosis 39(3): 212-24.

Pekala RJ, Wenger CF, Levine RL (1985) Individual differences in phenomenological experience: states of consciousness as a function of absorption. Journal of Personality and Social Psychology 48: $125-32$.

Perry C, Nadon R, Button J (1992) The measurement of hypnotic ability. In: E Fromm, MR Nash (eds) Contemporary Hypnosis Research, New York: Guilford, 459-90.

Peter B (2001) Hypnose und die Konstruktion von Wirklichkeit. In: D Revenstorf, B Peter (Hrsg) Hypnose in Psychotherapie und Psychosomatik und Medizin. Ein Manual für die Praxis. Heidelberg: Springer, S. 33-53.

Peters JP, Dhanens TP, Lundy RM, Landy FJ (1974) A factor analytic investigation of the Harvard Group Scale of Hypnotic Susceptibility, Form A. International Journal of Clinical and Experimental Hypnosis 22: 377-85. 
Plotnick AW, Paine PA, O’Grady DJ (1991) Correlates of hypnotizability in children: absorption, vividness of imagery, phantasy play, and social desirability. American Journal of Clinical Hypnosis 34 (1): 1-58.

Shor RE, Orne EC (1962) Harvard Group Scale of Hypnotic Susceptibility, Form A. Palo Alto, CA: Consulting Psychologists Press. (Deutsche Fassung von W. Bongartz, Universität Konstanz, 1982).

Shor RE, Orne EC (1963) Norms on the Harvard Group Scale of Hypnotic Susceptibility, Form A. International Journal of Clinical and Experimental Hypnosis 11: 39-48.

Spanos NP, Flynn DM, Gwynn MI (1989) Kontext-Anforderungen, negative Halluzinationen und die Geheime-Beobachter-Reaktion: Drei Geheime Beobachter beobachtet. Hypnose und Kognition 6: 33-40.

Spanos NP, Mah CD, Pawlak AE, D’Eon JL, Ritchie G (1980) A multivariate and factor analytic study of hypnotic susceptibility. Unpublished Manuscript, Carleton University, Ottawa, Ontario, Canada.

Stukat KG (1958) Suggestibility: A Factorial and Experimental Analysis. Stockholm: Almquist \& Wiksell.

Tellegen A (1978-79) On measures and conceptions of hypnosis. American Journal of Clinical Hypnosis 21(2\&3): 219-36.

Tellegen A, Atkinson G (1974) Openess to absorbing and self-altering experiences ('absorption'), a trait related to hypnotic susceptibility. Journal of Abnormal Psychology 83: 268-77.

Weitzenhoffer AM (1980) Hypnotic susceptibility revisited. American Journal of Clinical Hypnosis 22: $130-46$.

Weitzenhoffer AM (1993) Suggestibilität und Hypnose im zwanzigsten Jahrhundert. Hypnose und Kognition 10: 78-86.

Weitzenhoffer AH, Hilgard ER (1959) Stanford Hypnotic Susceptibility Scale, Forms A and B. Palo Alto, CA: Consulting Psychologists Press. (Deutsche Fassung von Walter Bongartz, University of Konstanz, 1982).

Woody EZ (1997) Have the hypnotic susceptibility scales outlived their usefulness? International Journal of Clinical and Experimental Hypnosis 45: 301-13.

Woody EZ, Bowers KS, Oakman JM (1992) A conceptual analysis of hypnotic responsiveness: experience, individual differences, and context. In: E Fromm, MR Nash (eds) Contemporary Hypnosis Research. New York: Guilford, 3-33.

Address for correspondence:

Dr Christoph Piesbergen

Department of Psychology

University of Munich

Leopoldstr. 13

80802 München

Germany

Email: chris.piesbergen@psy.uni-muenchen.de 